“C 2019 IEEE. Personal use of this material is permitted. Permission from IEEE must be obtained for all other uses, in any current or future media, including reprinting/republishing this material for advertising or promotional purposes, creating new collective works, for resale or redistribution to servers or lists, or reuse of any copyrighted component of this work in other works." 


\section{Localisation of the Lines of Response in a Continuous Cylindrical Shell PET Scanner}

\author{
Keenan J. Wilson, Roumani Alabd, \\ Mehran Abolhasan and Daniel R. Franklin* \\ School of Electrical and Data Engineering \\ University of Technology Sydney \\ Email: *Daniel.Franklin@uts.edu.au
}

\author{
Mitra Safavi-Naeini \\ Australia's Nuclear Science \& Technology Organisation
}

\begin{abstract}
This work presents a technique for localising the endpoints of the lines of response in a PET scanner based on a continuous cylindrical shell scintillator. The technique is demonstrated by applying it to a simulation of a sensitivityoptimised continuous cylindrical shell PET system using two novel scintillator materials - a transparent ceramic garnet, GLuGAG:Ce, and a $\mathrm{LuF}_{3}$ :Ce-polystyrene nanocomposite. Error distributions for the endpoints of the lines of response in the axial, tangential and radial dimension as well as overall endpoint spatial error are calculated for three source positions; the resultant distribution of error in the placement of the lines of response is also estimated.
\end{abstract}

Index Terms-PET, nanocomposite, ceramic, scintillator.

\section{INTRODUCTION}

One of the challenges in increasing the availability of clinical and research positron emission tomography (PET) is the substantial capital expense of the scanner. A major fraction of these costs is due to the scintillator crystals, used for the detection of coincident $511 \mathrm{keV}$ photons emitted from the annihilation of positrons within the subject [1][3]. There is growing interest in new scintillator materials with less-stringent manufacturing requirements and greater geometric flexibility compared to the discrete monocrystalline materials presently used in PET. These materials include polycrystalline ceramic garnets and scintillator-polymer nanocomposites. The lower cost of these materials must be weighed against their typically inferior physical properties, which include lower optical transmittivity and, in the case of nanocomposites, lower density and effective atomic number. A PET scanner utilising such scintillator materials therefore offers a fundamentally different set of engineering trade-offs compared to those employing monocrystalline scintillators.

The geometric flexibility offered by these new materials includes the potential for creating novel structures and geometries which would be difficult or expensive to fabricate with monocrystalline materials. One such geometry is a continuous monolithic cylindrical shell, with photodetectors tiled both on the inner and outer surface. In a monolithic scintillator, the optical photon distribution detected on one or both sides of the scintillating layer is used to estimate the 3D location of the endpoints of the line of response (LoR) between both halves of a coincidence pair. Compared to a traditional pixellated detector design, a continuous cylindrical shell offers numerous advantages, including uniform gapfree angular coverage around the centre of the field of view (FoV) and intrinsically high-resolution estimation of depth of interaction (DoI), which is expected to provide good uniformity of spatial resolution.

Several authors have proposed methods for localising the point of interaction of a gamma photon in a planar scintillator slab, including error-minimising optimisation approaches based on an analytic model of photon distribution within the slab, and neural network-based approaches which are adept at dealing with the non-uniformity of scintillator response near the edge of the slab [4]-[6]. In this paper, we describe an adaptation of these methods to a continuous cylindrical shell PET scanner, and present the first quantitative simulation results for a continuous-shell cylindrical PET system. A Monte Carlo-based technique for optimising the thickness of ceramic and nanocomposite scintillator materials for accurate localisation of detected gamma photons is applied to the two promising new scintillator materials. The results are used to design an approximately cylindrical-shell PET scanner with its shell thickness optimised to maximise probability of localisation of the point of interaction to within $5 \mathrm{~mm}$ of the true location, for one ceramic (GLuGAG:Ce) and one nanocomposite material $\left(\mathrm{LaF}_{3} / \mathrm{PS}\right)$. Simulations are performed using the open source Geant4 Application for Tomographic Emission (GATE; [7], [8]), with a point source positioned at three locations within the scanner field of view (at the centre of scanner, at an offset of $50 \%$ of the inner radius and at an offset of $75 \%$ of the inner radius). The error in locating the endpoints of the line of response in the radial, axial, tangential dimensions, together with the overall error and the error in placement of the line of response itself, is evaluated.

Section II briefly describes the optimisation method used to determine the scanner geometry; it also discusses the localisation technique and lists the key properties of the simulated scintillator materials. Section III presents the results of the simulation study, with the key implications discussed in Section IV. Section V summarises the findings from this work and outlines the next steps for this research project. 
TABLE I

SCINTILLATOR PROPERTIES. ALL VALUES ARE TAKEN FROM THE LITERATURE UNLESS INDICATED WITH *, IN WHICH CASE THE PARAMETER WAS THEORETICALLY CALCULATED BASED ON OTHER PREVIOUSLY-PUBLISHED MATERIAL PROPERTIES [13]-[15].

\begin{tabular}{lll}
\hline Material & GLuGAG:Ce & $\mathrm{LaF}_{3}: C e-P S$ \\
\hline Type & Ceramic & Nanocomposite \\
Peak $\lambda(\mathrm{nm})$ & 550 & 334 \\
Primary decay time $(\mathrm{ns})$ & 84 & 30 \\
Light yield $(\mathrm{ph} / \mathrm{MeV})$ & 48200 & 4500 \\
$\rho$ & 6.9 & 3.47 \\
$n\left(\lambda_{p}\right)$ & 1.92 & $1.65^{*}$ \\
\hline
\end{tabular}

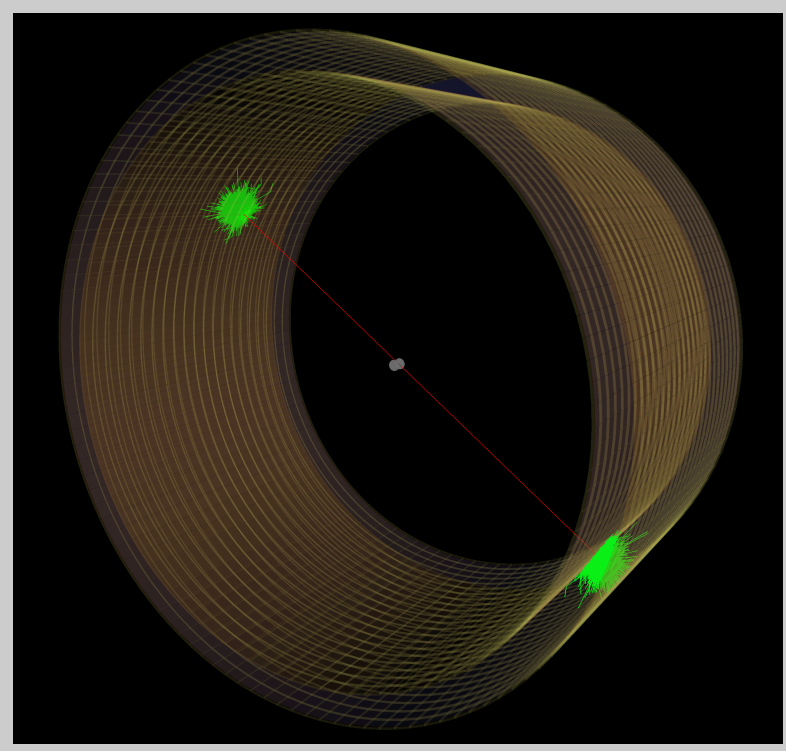

Fig. 1. Geometry of the simulated scanners (GLuGAG:Ce is shown; the approximated cylindrical shell is slightly thicker with $\mathrm{LaF}_{3}$-PS. Optical photons are shown in green.

\section{MATERIALS AND METHODS}

\section{A. Materials}

The scintillator materials used in this study have been identified as excellent candidates for monolithic scintillators in PET systems due to their combination of good optical transmittivity at the emission wavelength and moderate to high linear attenuation coefficient for $511 \mathrm{keV}$ gamma photons. GLuGAG:Ce is a synthetic garnet, which can be fabricated as a polycrystalline ceramic with similar physical properties to the crystalline form [9]. $\mathrm{LaF}_{3}$ :Ce-PS is one of the best-performing nanocomposite scintillator materials, since the close match between the refractive index of its constituent components results in good optical transmittivity at its emission wavelength, even with a high loading factor (50\% in this work) [10]-[12]. The key optical properties of the scintillators are listed in Table I.

\section{B. Optimisation of scanner geometry}

The scanner is designed for small animal or human head imaging. To allow the simulated photodetectors to be tiled to
TABLE II

SCANNER DIMENSIONS AND EXPECTED DETECTION EFFICIENCY

\begin{tabular}{lll}
\hline Material & GLuGAG:Ce & $\mathrm{LaF}_{3}: \mathrm{Ce}-\mathrm{PS}$ \\
\hline Length $(\mathrm{mm})$ & 296 & 296 \\
Inner radius $r_{\text {in }}(\mathrm{mm})$ & 233.3145 & 233.3145 \\
Inner faces & 104 & 104 \\
Inner face width $(\mathrm{mm})$ & 14.442 & 14.442 \\
Outer radius $r_{\text {out }}(\mathrm{mm})$ & 258.2385 & 276.1885 \\
Average thickness $(\mathrm{mm})$ & 24.919 & 42.874 \\
Outer faces & 116 & 124 \\
Outer face width & 14.2 & 14.2 \\
Detection efficiency $(\%)$ & 67.82 & 48.83 \\
Photoelectric fraction $(\%)$ & 24.33 & 9.95 \\
\hline
\end{tabular}

the inner and outer surface of the scintillating cylinder, the cylindrical shell is approximated as the difference between two polygonal prisms. The geometry is shown in Fig. 1. Each inner and outer face can accommodate an axial row of 21 square pixellated silicon detectors, each comprising a $14 \times 14$ array of $1 \mathrm{~mm} \times 1 \mathrm{~mm}$ pixels.

For each material, the specific thickness of the scintillator shell has been determined using an approach which maximises the efficiency of accurate endpoint localisation. In summary, a small number of Monte Carlo simulations are conducted in which $511 \mathrm{keV}$ photons were directed perpendicularly into a slab of the material under test, and localisation was performed by parametrically fitting an analytic model of the expected optical photon distribution to the observed photon maps (a simpler version of the method described in Section II-C). The probability of estimating the location of the point of interaction to within a threshold of $5 \mathrm{~mm}$ was computed, and a polynomial fit performed to identify the thickness which will maximise this probability.

For GLuGAG:Ce and $\mathrm{LaF}_{3}: \mathrm{Ce}-\mathrm{PS}$, this resulted in the average thicknesses listed in Table II; the expected detection efficiency (expressed as a percentage of incident normal $511 \mathrm{keV}$ gamma photons) and percentage of interactions which are purely photoelectric for the given dimensions is also listed.

\section{Localisation method}

The localisation technique described in this work assumes all optical photon events can be detected and timestamped with temporal resolution of the order of $10 \mathrm{~ns}$ or better (easily achievable with digital SIPM detectors [16]), and logged in list mode. The localisation method is a cylindrical-shell extension to the methods introduced by Li et al., which aim to fit an analytic model of optical photon distribution within a planar monolithic scintillator slab to the observed optical photon distribution [4], [5].

A suspected gamma interaction with the scintillator is considered for coincidence analysis if a spatio-temporal cluster of more than $N_{\min }$ optical photons are detected on both the inner and outer detector arrays $\left(N_{\min } \geq 10\right.$ is sufficient for satisfactory localisation for almost all detected events) within a timing window $\tau_{d}$ (inner and outer peak photon detection 
should be almost simultaneous; $\tau_{d}$ may be of the order of half the decay time constant of the scintillator). A potential coincidence is two double-sided clusters occurring within a coincidence timing window $\tau_{c}>\tau_{d}$ (in this work, $\tau_{d}$ is set to the primary decay time constant of the scintillator, which is much greater than the maximum cross-scanner time of flight of approximately $1.84 \mathrm{~ns}$ ). Fig. 1 shows a typical detected coincidence and the resulting showers of optical photons.

The polar coordinates $\left(r_{\text {out }}, \theta_{\text {out }}, z\right)$ of each pixel on the outer surface are converted to Cartesian coordinates $\left(r_{\text {out }}, y=r_{\text {out }} \theta_{\text {out }}, z\right)$, where $z$ and $y$ are the axial and tangential displacements, respectively. The polar coordinates of each pixel on the inner surface are similarly converted to Cartesian coordinates, with $y=r_{\text {out }} \theta_{\text {in }}$ scaled using the outer radius such that the cylindrical shell is 'unrolled' into a rectangular slab in which both inner and outer surfaces have the same tangential width. The computed $y$ and $z$ coordinates of each pixel are stored in a pair of look-up tables.

A coincidence event will appear as two approximately simultaneous clusters of nonzero pixels, with cluster peaks separated by a minimum angle related to the maximum size of the subject. Clusters will not physically overlap unless the coincidence is due to randoms or scattering of an emitted photon; such cases are rejected. The localisation algorithm requires the cylindrical shell to be cut in two places to separate each half of the coincidence pair. To do this, the inner and outer pixel arrays are first added together, then summed axially, resulting in a one-dimensional signal featuring two peaks. The higher of the two peaks is first located, and then excised from the 1D signal based on the maximum theoretical radius of the cluster. Then, the smaller peak is located in the residual signal. The unrolled pixel maps are then cut half-way between the smaller and larger angles separating these two peaks, resulting in a pair of two-sided images of the pixel clusters. The size of each of these arrays is reduced by shrinking a rectangular region around each cluster, excluding all contiguous rectangular regions with no optical photon detections from the arrays. The remaining cluster regions and their corresponding axial and tangential coordinates are then passed to the fitting algorithm.

Analytic expressions for the expected inner and outer photon distributions are given by the attenuated Cauchy distributions in (1) and (2), respectively.

$$
\begin{aligned}
& J_{o}\left(z_{d}, y_{d}\right)=
\end{aligned}
$$

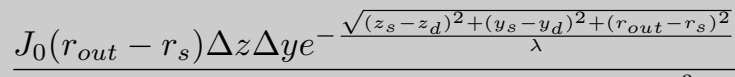

$$
\begin{aligned}
& 4 \pi\left(\left(z_{s}-z_{d}\right)^{2}+\left(y_{s}-y_{d}\right)^{2}+\left(r_{\text {out }}-r_{s}\right)^{2}\right)^{\frac{3}{2}} \\
& J_{i}\left(z_{d}, y_{d}\right)= \\
& \frac{J_{0}\left(r_{s}-r_{i n}\right) \Delta z \Delta y e^{-\frac{\sqrt{\left(z_{s}-z_{d}\right)^{2}+\left(y_{s}-y_{d}\right)^{2}+\left(r_{s}-r_{i n}\right)^{2}}}{\lambda}}}{4 \pi\left(\left(z_{s}-z_{d}\right)^{2}+\left(y_{s}-y_{d}\right)^{2}+\left(r_{s}-r_{i n}\right)^{2}\right)^{\frac{3}{2}}}
\end{aligned}
$$

where $y_{d}$ and $z_{d}$ are the a point on the respective detector surfaces, the point of scintillation is $\left(r_{s}, y_{s}, z_{s}\right), \Delta z$ and $\Delta y$ are the (scaled) pixel dimensions, $J_{0}$ is the number of emitted scintillation photons, $\lambda$ is the radiation attenuation length, and $r_{i n}$ and $r_{\text {out }}$ are the inner and outer radius of the cylindrical shell, respectively.

To account for total internal reflection within the scintillator slab ${ }^{1}, J_{b}$ is set to zero anywhere outside a circle of radius

$$
R_{o}=z_{s} \frac{n_{m m}}{n_{s}^{2}-n_{m m}^{2}}
$$

where $n_{m m}$ is the refractive index of Meltmount (the optical coupling compound) and $n_{s}$ is the scintillator's refractive index. Similarly $J_{f}$ is set to zero outside of a circle of radius

$$
R_{i}=\left(T-z_{s}\right) \frac{n_{m m}}{n_{s}^{2}-n_{m m}^{2}}
$$

The estimated coordinates of the endpoints of the line of response are calculated by jointly minimising the error between (1) and (2) and the observed photon map using the Levenberg-Marquardt algorithm. Finally, the endpoints of the LoR are converted back into cylindrical coordinates and logged.

Energy windowing is straightforward with this detection model as the number of optical photons emitted by the interaction may be estimated from the observed photon maps; detection of scattered photons may be discarded if this number is below an arbitrary threshold.

\section{Simulation}

GATE 8.1/Geant4 10.4p02 are used for all simulations. GATE has been built with full optical photon tracking turned on. Optical properties of the materials are based on values obtained from the literature [9]-[12]. The scintillator ring is optically coupled to the array of ideal silicon photodetectors with Meltmount optical epoxy [17].

A cylindrical ${ }^{18} \mathrm{~F}$ source (radius $=1 \mathrm{~mm}$, height $=2 \mathrm{~mm}$ ) is placed at the centre of the field of view, encased in a cylindrical water phantom (radius $=5 \mathrm{~mm}$, height $=10 \mathrm{~mm}$ ). Simulations have been conducted with 50000 decays for each scintillator material. For each interaction with the scintillator, the true coordinates of the endpoints were logged together with the coordinates of each interaction of the optical photons with the photodetectors. The optical photon coordinates are rebinned to $1 \mathrm{~mm} \times 1 \mathrm{~mm}$ pixels and processed with the coincidence logic, and the error in each dimension together with the overall error for accuracy of endpoint estimation computed. Finally, the distance of the LoR from the centre of the true source location is computed for each detected coincidence.

${ }^{1}$ Only a small minority of scintillation photons are internally reflected; most of these are absorbed by the scintillator before reaching a detector. 


\section{RESULTS}

The accuracy of LoR endpoint localisation is visualised using hexagonal-cell heatmaps of the error scatterplots in the radial, axial and rotational dimensions, plus an overall error and the error in placement of the line of response, as a function of increasing depth of interaction for both evaluated materials.

\section{A. GLuGAG:Ce}

Fig. 2(a), 2(b) and 2(c) illustrate the errors in each dimension for GLuGAG:Ce. Overall error in endpoint location is shown in Fig. 2(d), while Fig. 3 shows the minimum distance from the estimated LoR to the nominal centre of the radioactive source. Results for the offset source are very similar.

A summary of the statistical range of the errors (first quartile, median and third quartile) for GLuGAG:Ce with the source at the centre and at radial offsets of $50 \%$ and $75 \%$ of the inner radius of the scintillator shell are presented in Table III. An additional metric is introduced in the table; total spatial error $(\Delta d)$.

\section{B. $\mathrm{LaF}_{3}: \mathrm{Ce}-\mathrm{PS}$}

Fig. 4(a), 4(b) and 4(c) illustrate the error in radial, axial and rotational dimensions for $\mathrm{LaF}_{3}$ :Ce-PS. Overall error in endpoint location is shown in Fig. 4(d), while Fig. 5 shows the minimum distance from the estimated LoR to the nominal centre of the radioactive source. Again, results for the offset source location are very similar.

A summary of the statistical range of the errors (first quartile, median and third quartile) for $\mathrm{LaF}_{3}$ :Ce-PS with the source in the same three positions (centre and offset at $50 \%$ and $75 \%$ of inner shell diameter) is presented in Table IV. Statistics for the total spatial error $(\Delta d)$ have also been included.

\section{Detector Sensitivity and Detection Accuracy}

Table $\mathrm{V}$ presents a summary of the overall sensitivity of the two designs for each source position, expressed as a fraction of positron annihilations which result in detection of both endpoints, and the fraction of estimated endpoint locations which are within $2 \mathrm{~mm}$ and $5 \mathrm{~mm}$ of their ground-truth locations.

\section{Discussion}

There are some significant differences between the behaviour of the two scintillator materials. The high density of GLuGAG:Ce results in most photons stopping within the first $10 \mathrm{~mm}$ of the material, whereas they penetrate more deeply into the $\mathrm{LaF}_{3}: \mathrm{Ce}-\mathrm{PS}$. For $\mathrm{LaF}_{3}$ :Ce-PS, all error metrics have quite long-tailed distributions - particularly in the $\Delta$ LoR metric. The largest component of the error is from the estimation of the depth of interaction in the cylindrical shell. Despite this, more than half of the detected gamma photons are localised in depth to an accuracy of better than $5 \mathrm{~mm}$ for $\mathrm{LaF}_{3}$ :Ce-PS and $2 \mathrm{~mm}$ for GLuGAG:Ce (Table
V) (with little variation with respect to radial offset), which compares well with most depth-of-interaction capable PET scanners of this size. Overall sensitivity of both variants of scanner geometry is very high, due to a combination of high scintillator thickness and geometric efficiency.

A large part of the error is due to the implicit assumption that all detected events are purely photoelectric, which is not true in practice. For both materials, our simulations show that more than three quarters of all interactions between incident gamma photons and the scintillator will be Comptonscattered, some more than once (see Table II). However, some of the scattered photons go on to entirely escape the scintillator, while the subsequent interactions of many others often occur close to the initial interaction. In these cases, if a non-trivial amount of energy is deposited, the fitting process will still work very well, since the total energy deposition is one of the degrees of freedom of the optimiser. For multiCompton interactions, since scattering is biased towards the forward direction, it is more probable than not that further interactions with the scintillator occur more deeply in the scintillator shell, leading to more optical attenuation of the resulting scintillation photons than for those produced during the initial interaction. This has results in observed optical photon pattern being typically dominated by the first and shallowest interaction. The parametric fit to the analytic model is therefore quite similar to the pure photoelectric case in many instances.

As expected for a PET system with high resolution DoI estimation capabilities, degradation in localisation performance with increased radial displacement is quite moderate, with the median error in LoR placement increasing from $1.27 \mathrm{~mm}$ to $1.62 \mathrm{~mm}$ between the centre and $75 \%$ of the inner radius of the cylindrical shell for GLuGAG:Ce, and $3.12 \mathrm{~mm}$ to $4.13 \mathrm{~mm}$ in the case of $\mathrm{LaF}_{3}$ :Ce-PS.

A direct comparison of the two materials suggests that GLuGAG:Ce is the superior material. However, this does not account for economic factors which also influence scanner design. The nanocomposite material has many desirable physical and mechanical properties. If the performance is adequate for the targeted application, it may very well be the more attractive material.

\section{CONCLUSIONS AND FUTURE WORK}

This work established the feasibility of a continuous cylindrical shell PET scanner based on either optically transparent ceramic garnet or inorganic scintillator/polymer nanocomposite materials. Initial simulations for a point source located at three positions within the scanner's field of view are presented, yielding promising results for the accuracy of detection of the endpoints of the line of response.

In the next phase of this study, we will perform detailed PET performance characterisation using the NEMA NU42008 protocol, and perform image reconstructions with point sources, line sources and phantoms at a variety of locations within the scanner's field of view. The median LoR displacement errors of 1.27-1.62 mm for GLuGAG:Ce 


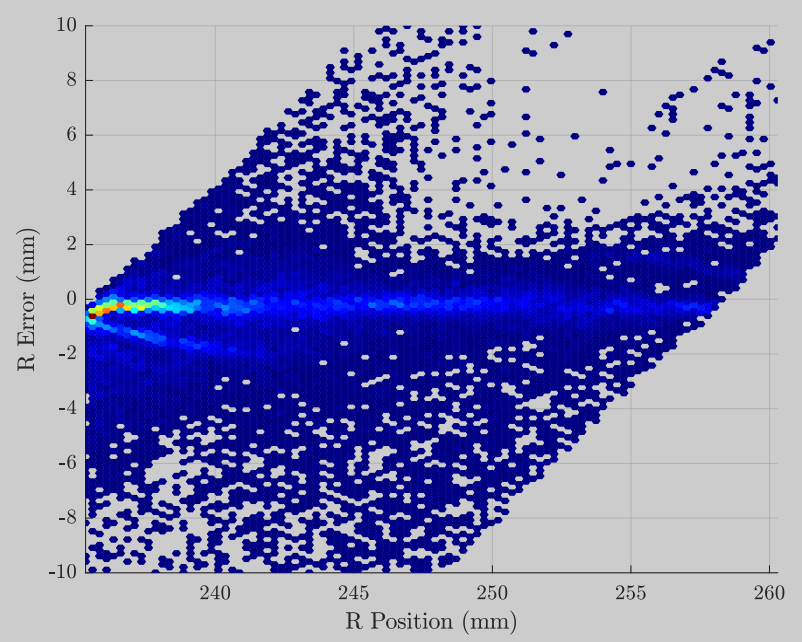

(a) Radial endpoint error

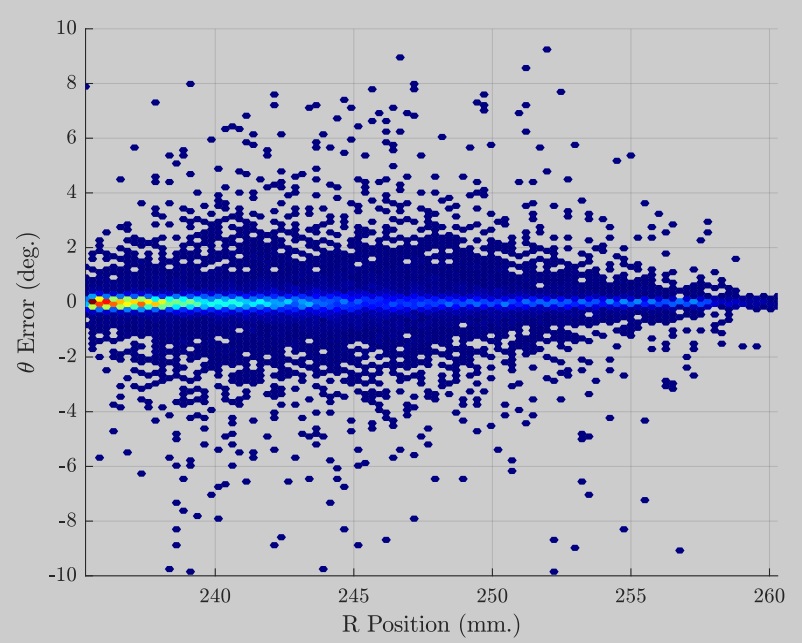

(c) Rotational endpoint error

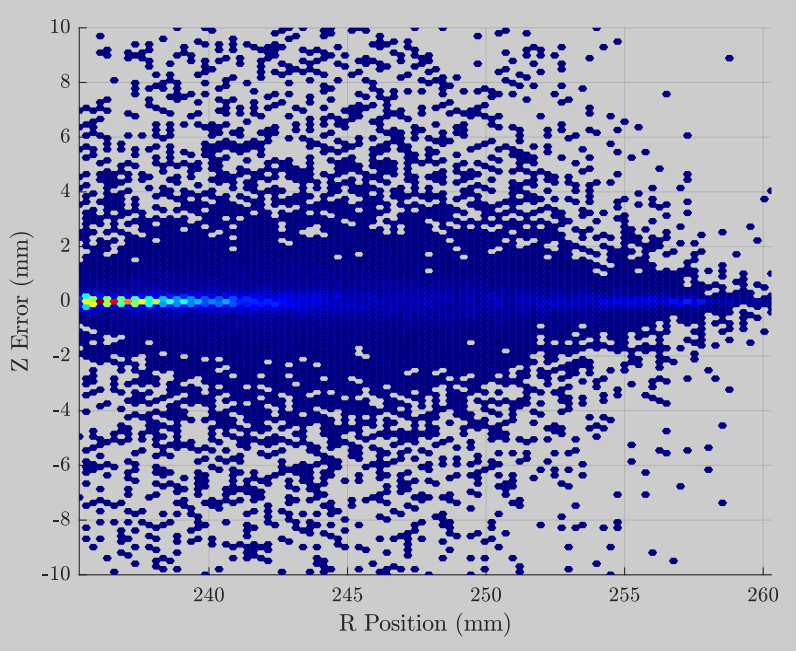

(b) Axial endpoint error

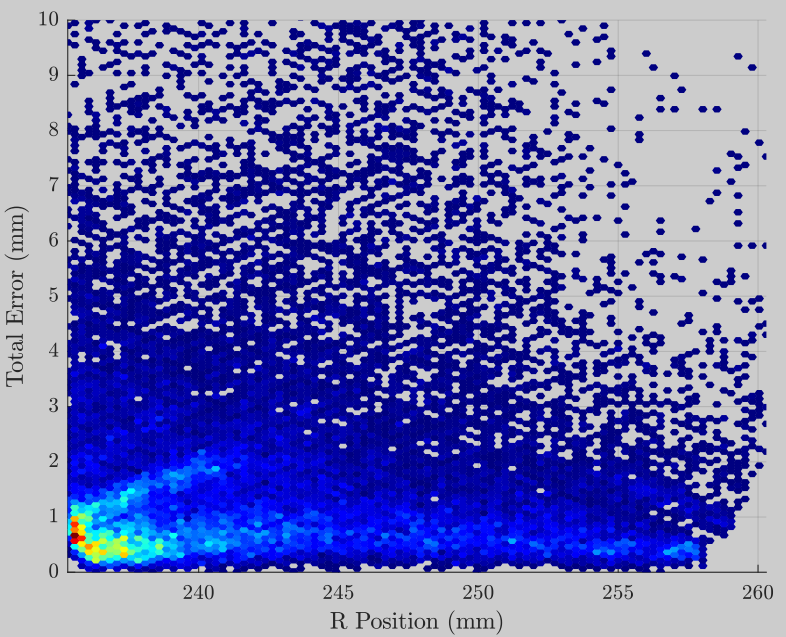

(d) Total endpoint error

Fig. 2. GLuGAG:Ce: endpoint errors in each dimension and overall vs. depth of penetration.

TABLE III

GLUGAG:CE ERROR STATISTICS SUMMARY

\begin{tabular}{|c|c|c|c|c|c|c|c|c|c|}
\hline \multirow{2}{*}{ Error } & \multicolumn{3}{|c|}{ Source Centre } & \multicolumn{3}{|c|}{ Source $50 \%$ Inner Radius } & \multicolumn{3}{|c|}{ Source $75 \%$ Inner Radius } \\
\hline & Q1 & Median & Q3 & Q1 & Median & Q3 & Q1 & Median & Q3 \\
\hline$\Delta \theta$ (Deg.) & -0.15 & -0.0082 & 0.13 & -0.14 & -0.0063 & 0.13 & -0.15 & -0.0074 & 0.13 \\
\hline$\Delta \mathrm{r}(\mathrm{mm})$ & -1.66 & -0.40 & 0.02 & -1.66 & -0.40 & 0.02 & -1.64 & -0.40 & 0.02 \\
\hline$\Delta \mathrm{z}(\mathrm{mm})$ & -0.29 & 0.0009 & 0.29 & -0.29 & 0.0003 & 0.29 & -0.29 & -0.00001 & 0.27 \\
\hline$\Delta \mathrm{d}(\mathrm{mm})$ & 0.68 & 1.40 & 3.44 & 0.68 & 1.42 & 3.62 & 0.68 & 1.42 & 3.91 \\
\hline$\Delta \mathrm{LoR}(\mathrm{mm})$ & 0.60 & 1.27 & 4.17 & 0.71 & 1.48 & 5.18 & 0.77 & 1.62 & 6.59 \\
\hline
\end{tabular}


TABLE IV

$\mathrm{LAF}_{3}$ :CE-PS ERROR STATISTICS SUMMARY

\begin{tabular}{|c|c|c|c|c|c|c|c|c|c|}
\hline \multirow{2}{*}{ Error } & \multicolumn{3}{|c|}{ Source Centre } & \multicolumn{3}{|c|}{ Source $50 \%$ Inner Radius } & \multicolumn{3}{|c|}{ Source $75 \%$ Inner Radius } \\
\hline & Q1 & Median & Q3 & Q1 & Median & Q3 & Q1 & Median & Q3 \\
\hline$\Delta \theta$ (Deg.) & -0.26 & -0.0019 & 0.25 & -0.27 & -0.0038 & 0.26 & -0.28 & -0.0029 & 0.27 \\
\hline$\Delta \mathrm{r}(\mathrm{mm})$ & -3.07 & 0.33 & 1.22 & -3.06 & -0.33 & 1.21 & -2.84 & -0.31 & 1.11 \\
\hline$\Delta \mathrm{z}(\mathrm{mm})$ & -0.92 & -0.0050 & 0.93 & -0.93 & 0.0038 & 0.99 & -1.02 & -0.0030 & 0.97 \\
\hline$\Delta \mathrm{d}(\mathrm{mm})$ & 1.35 & 3.52 & 8.33 & 1.34 & 3.57 & 9.26 & 1.23 & 3.49 & 10.87 \\
\hline$\Delta \mathrm{LoR}(\mathrm{mm})$ & 1.22 & 3.12 & 11.78 & 1.43 & 3.59 & 16.60 & 1.50 & 4.13 & 24.10 \\
\hline
\end{tabular}

TABLE V

FRACTIONS OF DECAYS YIELDING USABLE LORS (BOTH DETECTED AS A PERCENTAGE OF TOTAL POSITRON ANNIHILATIONS) AND ACCURACY OF DETECTED ENDPOINTS AS A PERCENTAGE DETECTED WITHIN THE SPECIFIED ERROR MARGIN.

\begin{tabular}{|c|c|c|c|c|c|c|}
\hline \multirow[b]{2}{*}{ Source Location } & \multicolumn{2}{|c|}{ Sensitivity (\%) } & \multicolumn{2}{|c|}{$\%<2 \mathrm{~mm}$ error } & \multicolumn{2}{|c|}{$\%<5 \mathrm{~mm}$ error } \\
\hline & GLuGAG:Ce & LaF3:Ce-PS & GLuGAG:Ce & LaF3:Ce-PS & GLuGAG:Ce & LaF3:Ce-PS \\
\hline Centre & 29 & 22 & 61.82 & 33.49 & 81.60 & 62.14 \\
\hline $50 \%$ Inner Radius & 28 & 21 & 61.22 & 33.62 & 80.43 & 60.91 \\
\hline $75 \%$ Inner Radius & 31 & 24 & 60.71 & 35.07 & 78.79 & 60.14 \\
\hline
\end{tabular}
0.75 in $19.1 \mathrm{~mm}$

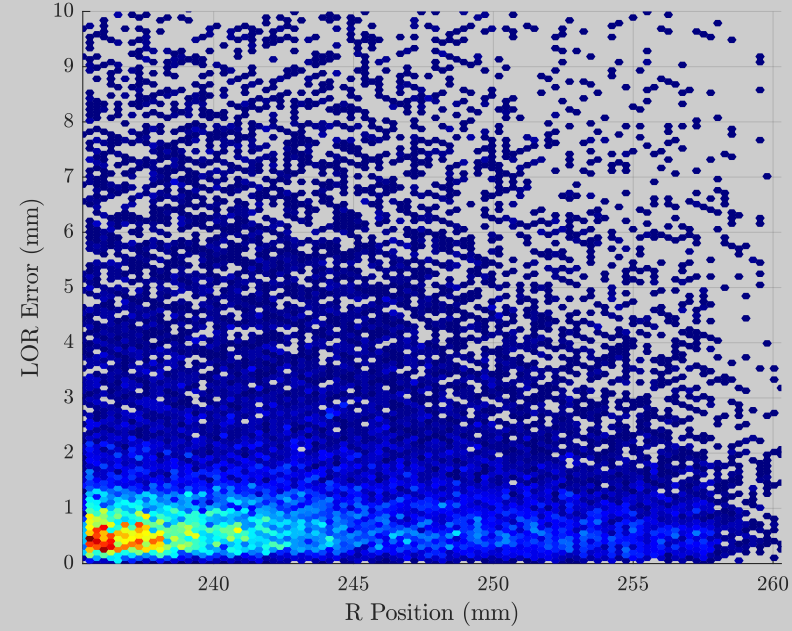

Fig. 3. GLuGAG:Ce: LoR offset error vs. depth of interaction

and 3.12-4.13 $\mathrm{mm}$ for $\mathrm{LaF}_{3}:$ Ce-PS obtained in this study are suggestive of good performance in the next phase of the project.

\section{ACKNOWLEDGEMENTS}

This work is supported by the Australian Government Research Training Program Scholarship and the ARCLab facility at UTS.

\section{REFERENCES}

[1] S. Surti, M. E. Werner, and J. S. Karp, "Study of PET scanner designs using clinical metrics to optimize the scanner axial FOV and crystal thickness," Physics in Medicine and Biology, vol. 58, no. 12, pp. 39954012, May 2013.
[2] S. Surti and J. S. Karp, "Impact of detector design on imaging performance of a long axial field-of-view, whole-body PET scanner," Physics in Medicine and Biology, vol. 60, no. 13, pp. 5343-5358, Jun 2015.

[3] S. R. Cherry, T. Jones, J. S. Karp, J. Qi, W. W. Moses, and R. D. Badawi, "Total-body PET: Maximizing sensitivity to create new opportunities for clinical research and patient care," Journal of Nuclear Medicine, vol. 59, no. 1, pp. 3-12, Sep. 2017.

[4] Z. Li, M. Wedrowski, P. Bruyndonckx, and G. Vandersteen, "Nonlinear least-squares modeling of 3D interaction position in a monolithic scintillator block," Physics in Medicine \& Biology, vol. 55, no. 21 , p. 6515, 2010.

[5] J. Cabello, A. Etxebeste, G. Llosá, and S. I. Ziegler, "Simulation study of PET detector limitations using continuous crystals," Physics in Medicine \& Biology, vol. 60, no. 9, pp. 3673-3694, 2015.

[6] P. Conde, A. Iborra, A. Gonzalez, L. Hernández, P. Bellido, L. Moliner J. Rigla, M. Rodríguez-Álvarez, F. Sánchez, M. Seimetz, A. Soriano, L. F. Vidal, and J. M. Benlloch, "Determination of the interaction position of gamma photons in monolithic scintillators using neural network fitting," IEEE Transactions on Nuclear Science, vol. 63, no. 1, pp. 30-36, 2016.

[7] S. Jan, G. Santin, D. Strul, S. Staelens, K. Assie, D. Autret, S. Avner, R. Barbier, M. Bardies, P. Bloomfield et al., "Gate: a simulation toolkit for PET and spect," Physics in Medicine \& Biology, vol. 49, no. 19, pp. $4543-4561,2004$

[8] S. Jan, D. Benoit, E. Becheva, T. Carlier, F. Cassol, P. Descourt, T. Frisson, L. Grevillot, L. Guigues, L. Maigne et al., "Gate v6: a major enhancement of the gate simulation platform enabling modelling of CT and radiotherapy," Physics in Medicine \& Biology, vol. 56, no. 4, pp. 881-901, 2011.

[9] Y. Wang, G. Baldoni, C. Brecher, W. H. Rhodes, U. Shirwadkar, J. Glodo, I. Shah, and C. Ji, "Properties of transparent (Gd, Lu)3(Al, Ga)5O12:Ce ceramic with $\mathrm{Mg}, \mathrm{Ca}$ and Ce co-dopants," in Medical Applications of Radiation Detectors V, H. B. Barber, L. R. Furenlid, and H. N. Roehrig, Eds. SPIE, Aug. 2015.

[10] R. K. Feller, G. M. Purdy, D. Ortiz-Acosta, S. Stange, A. Li, E. A McKigney, E. I. Esch, R. E. Muenchausen, R. Gilbertson, M. Bacrania, B. L. Bennett, K. C. Ott, L. Brown, C. S. Macomber, B. L. Scott, and R. E. Del Sesto, "Large-scale synthesis of CexLa1-xF3 nanocomposite scintillator materials," Journal of Materials Chemistry, vol. 21, no. 15 , pp. 5716-5722, 2011.

[11] R. E. Del Sesto, E. A. McKigney, D. W. Cooke, R. E. Muenchausen, K. C. Ott, R. D. Gilbertson, T. M. McCleskey, M. Bacrania, L. G.

\section{$54 \mathrm{pt}$} 0.75 in $19.1 \mathrm{~mm}$ 


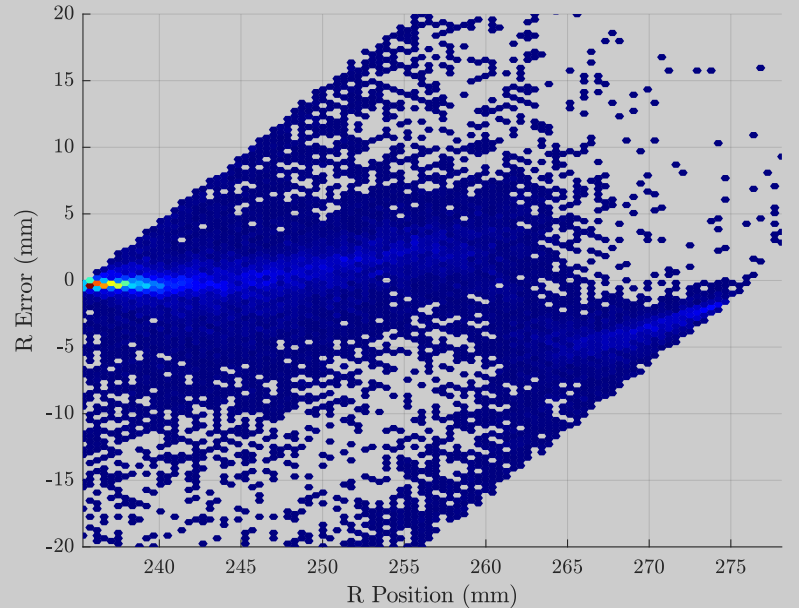

(a) Radial endpoint error

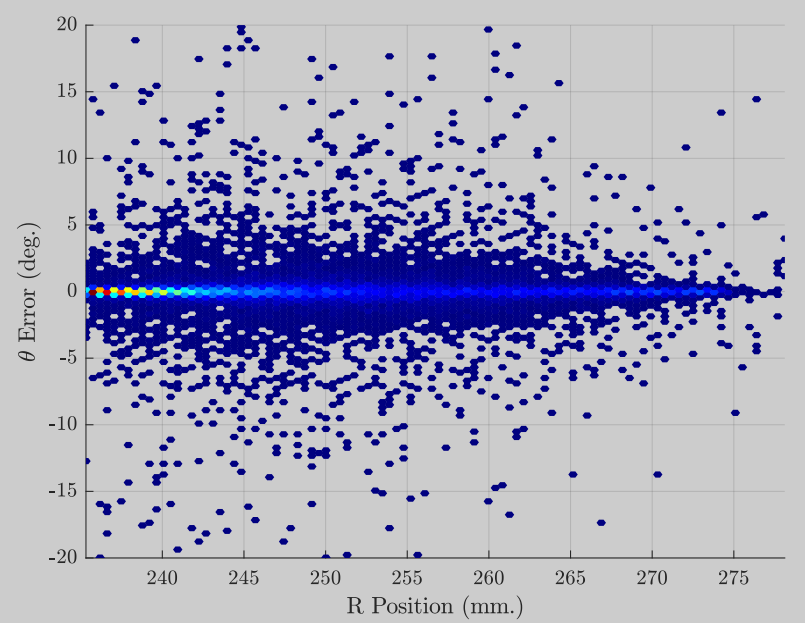

(c) Rotational endpoint error

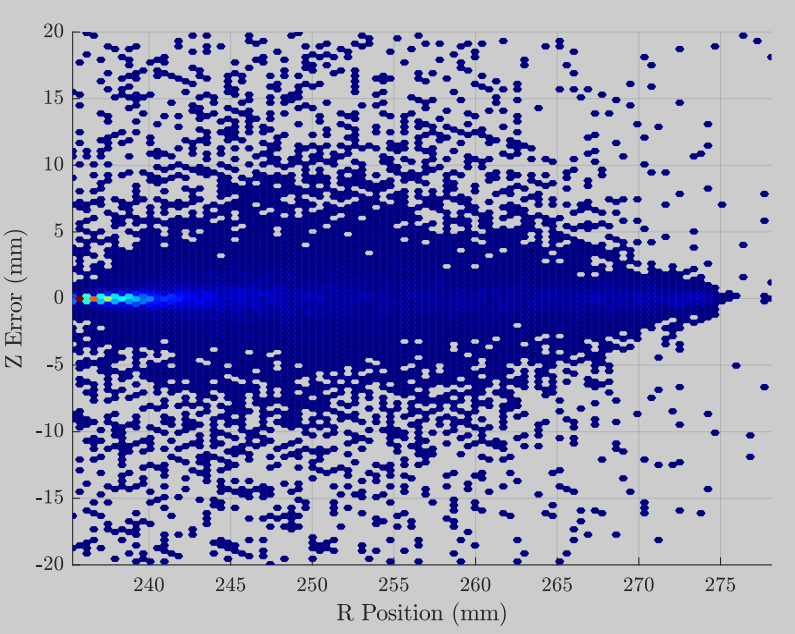

(b) Axial endpoint error

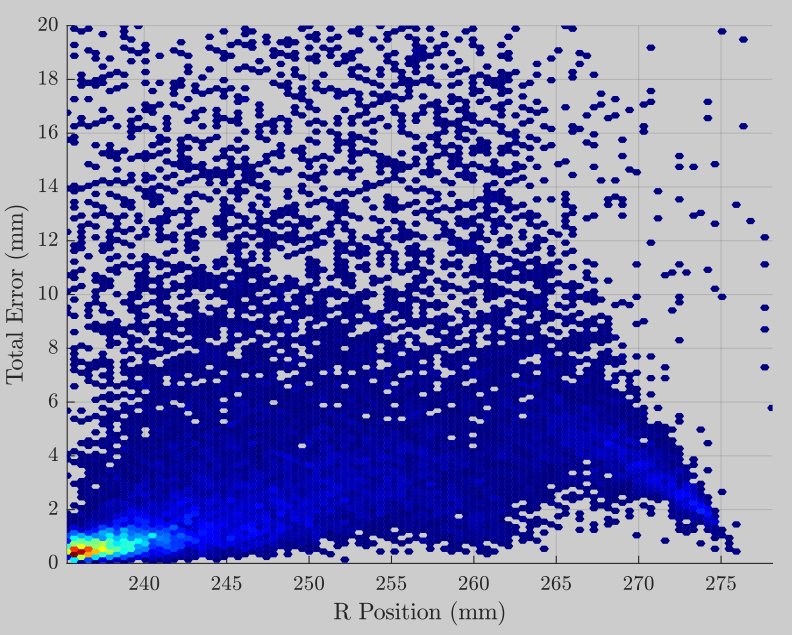

(d) Total endpoint error

Fig. 4. $\mathrm{LaF}_{3}$ :Ce-PS: endpoint errors in each dimension and overall vs. depth of penetration.

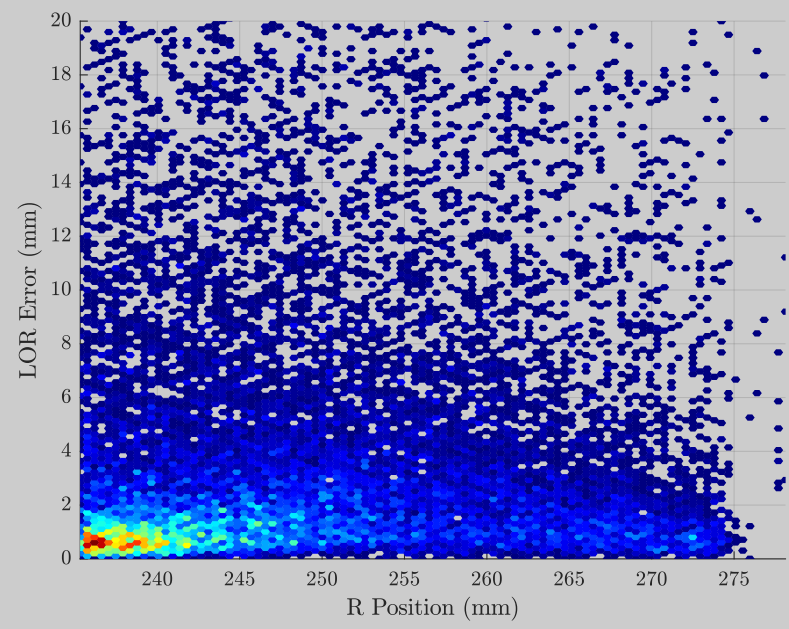

Fig. 5. $\mathrm{LaF}_{3}$ :Ce-PS: LoR offset error vs. depth of penetration

Jacobsohn, A. K. Burrell, B. L. Bennett, S. C. Sitarz, and J. F. Smith, "Development of nanocomposite scintillators," 2007.
[12] M. Osiński, J. B. Plumley, N. J. Withers, A. C. Rivera, B. A Akins, K. Sankar, and G. A. Smolyakov, "Lanthanide-halide-based nanoscintillators for portable radiological detectors," in Optics and Photonics in Global Homeland Security V and Biometric Technology for Human Identification VI, ser. Proceedings of SPIE, vol. 7306, May 2009, p. 730617.

[13] W. Caseri, "Nanocomposites," in The Chemistry of Nanostructured Materials, P. Yang, Ed. Singapore: World Scientific Publishing Co. Pte. Ltd., 2003, ch. 13, pp. 359-386.

[14] D. Szabó and T. Hanemann, "Polymer nanocomposites for optical applications," in Advances in Polymer Nanocomposites - Types and Applications, F. Gao, Ed. Cambridge: Woodhead Publishing Ltd., 2012, ch. 17, pp. 567-604.

[15] T. Kyprianidou-Leodidou, W. Caseri, and U. W. Suter, "Size variation of PbS particles in high-refractive-index nanocomposites," The Journal of Physical Chemistry, vol. 98, no. 36, pp. 8992-8997, 1994.

[16] S. Seifert, G. van der Lei, H. T. van Dam, and D. R. Schaart, "First characterization of a digital SiPM based time-of-flight PET detector with $1 \mathrm{~mm}$ spatial resolution," Physics in Medicine and Biology, vol. 58, no. 9, pp. 3061-3074, Apr. 2013.

[17] D. J. van der Laan, D. R. Schaart, M. C. Maas, F. J. Beekman, P. Bruyndonckx, and C. W. van Eijk, "Optical simulation of monolithic scintillator detectors using gate/geant4," Physics in Medicine \& Biology, vol. 55, no. 6, p. 1659, 2010. 